\title{
3 (IN)SATISFAÇÃO COM A IMAGEM CORPORAL E ATITUDES ALIMENTARES EM ESTUDANTES DO ENSINO SECUNDÁRIO
}

\author{
| Maria de Lurdes Pires'; Adília Fernandes²; Ana Maria Pereira ${ }^{3} \mid$
}

\section{RESUMO}

CONTEXTO: A autoimagem é um conceito transversal a toda humanidade, muito ligada à identidade pessoal. A preocupação permanente com a imagem corporal, leva a que os jovens procurem alcançar a aparência física perfeita, podendo adotar atitudes alimentares disfuncionais, com implicações na sua saúde.

OBJETIVOS: Avaliar as atitudes alimentares e a satisfação com a imagem corporal em estudantes do ensino secundário.

MÉTODOS: Estudo observacional, analítico e transversal. Foram avaliados 184 alunos aos quais foi aplicado um questionário que incluía o Questionário de Silhuetas de Collins e a escala Children's Eating Attitude Test validada para a população Portuguesa. Os dados foram tratados recorrendo-se ao programa SPSS, versão 22.0.

RESULTADOS: Verificou-se que todas as dimensões da Children's Eating Attitude Test apresentavam valores médios baixos traduzindo-se em comportamentos alimentares normais. A aplicação do Questionário de Silhuetas de Collins revelou que 38\% dos adolescentes estava satisfeito com a sua imagem corporal. Os resultados da escala de Children’s Eating Attitude Test em função da satisfação com a imagem corporal indicam que as atitudes alimentares são estatisticamente distintas entre os jovens satisfeitos e não satisfeitos com a silhueta, especificamente nas dimensões cumprimento da dieta $(\mathrm{p}=0,004)$ e controlo da ingestão alimentar $(\mathrm{p}=0,033)$.

CONCLUSÕES: Os resultados obtidos revelam a predominância de insatisfação com a imagem corporal dos jovens, assim como a existência de relação entre as atitudes alimentares e a satisfação corporal. Realça-se a necessidade de implementar estratégias multidisciplinares, não apenas direcionadas aos jovens, mas alargadas à dinâmica familiar.

\section{PALAVRAS-CHAVE: Alimentação; Imagem corporal; Estudantes}

\section{RESUMEN}

“(In)satisfacción con la imagen corporal y actitudes alimentarias en estudiantes de la enseñanza secundaria”

CONTEXTO: La autoimagen es un concepto transversal a toda la humanidad, muy ligada a la identidad personal. La preocupación permanente con la imagen corporal, lleva a que los jóvenes procuren alcanzar la apariencia física perfecta, pudiendo adoptar actitudes alimentarias disfuncionales, con implicaciones en su salud.

OBJETIVOS: Evaluar las actitudes alimenticias y la satisfacción con la imagen corporal en estudiantes de enseñanza secundaria.

METODOLOGÍA: Estudio observacional, analítico y transversal. Se evaluaron 184 estudiantes quienes se les aplicó un formulario que incluía el Cuestionario Siluetas Collins y la escala Children's Eating Attitude Test validado para la población portuguesa. Los datos fueron tratados utilizando el programa SPSS, versión 22.0.

RESULTADOS: Se verificó que todas las dimensiones de la Children's Eating Attitude Test scale presentaban valores medios bajos, traduciéndose en comportamientos alimentarios normales. La aplicación del Cuestionario de Siluetas de Collins reveló que $38 \%$ de los adolescentes estaba satisfecho con su imagen corporal. Los resultados de la escala de Children's Eating Attitude Test scale en función de la satisfacción con la imagen corporal indican que las actitudes alimenticias son estadísticamente distintas entre los jóvenes satisfechos y no satisfechos con la silueta, específicamente en las dimensiones cumplimiento de la dieta $(\mathrm{p}=0,004)$ y control de la ingesta alimentaria ( $\mathrm{p}$ $=0,004) \mathrm{p}=0,033)$.

CONCLUSIONES: Los resultados obtenidos revelan la predominancia de insatisfacción con la imagen corporal de los jóvenes, así como la existencia de relación entre las actitudes alimenticias y la satisfacción corporal. Se destaca la necesidad de implementar estrategias multidisciplinares, no sólo dirigidas a los jóvenes, sino que se amplían a la dinámica familiar.

DESCRIPTORES: Alimentación; Imagen corporal; Estudiantes

\begin{abstract}
“(Dis)satisfaction with corporal image and alimentary attitudes in high school students"

BACKGROUND: Self-image is a transversal concept to all humanity, closely related to personal identity. The ongoing concern with self-image causes young people to try to reach the perfect physical appearance, and they may adopt dysfunctional alimentary attitudes with effects on their health.

AIM: To evaluate the alimentary attitudes and satisfaction with corporal image in high school students.

METHODS: Observational, analytic and transversal study. 184 students were evaluated through an application form that included the Collins and the Children's Eating Attitude Test scale validated for Portuguese population. The data was treated using the SPSS programme, 2.0 version.

RESULTS: It was verified that all Children's Eating Attitude Test scale dimension presented low medium value translated in normal nutritional habits. The application of the Collins Questionnaire has revealed that $38 \%$ of the teenagers was satisfied with their body image. The results from the Children's Eating Attitude Test s scale according to the satisfaction with body image indicate that alimentary attitudes statistically different between the satisfied and non-satisfied young with their silhouette, specifically in the dimensions of diet compliance $(\mathrm{p}=0,004)$ and dietary intake control $(\mathrm{p}=0,033)$.

CONCLUSIONS: The obtained results reveal the predominance of dissatisfaction with body image of young people, as well as the existence of a relation between the alimentary attitudes and corporal satisfaction. It is necessary to implement multidisciplinary strategies, not only directed to the young, but also the family dynamic.
\end{abstract}

\section{KEYWORDS: Feeding; Body image; Students}

Submetido em 30-12-2018

Aceite em 26-03-2019

\footnotetext{
1 Mestre; Enfermeira especialista em Enfermagem Comunitária; Enfermeira no Hospital de Dia da Unidade Local de Saúde do Nordeste, E.P.E., Unidade Hospitalar Bragança, Avenida Abade Baçal, 5301-852 Bragança, Portugal, nuspires@gmail.com

2 Doutora; Investigadora na Health Sciences Research Unit: Nursing (UICISA: E); Professora Adjunta no Instituto Politécnico de Bragança, Campus de Santa Apolónia, Departamento de Enfermagem, 5300-253 Bragança, Portugal, adilia@ipb.pt

3 Doutora; Professora Adjunta no Instituto Politécnico de Bragança, Campus de Santa Apolónia, Escola Superior de Saúde de Bragança, Departamento de Tecnologias de Diagnóstico e Terapêutica, Centro de Investigação de Montanha, 5300-253 Bragança, Portugal, amgpereira@ipb.pt

Citação: Pires, M. L., Fernandes, A., \& Pereira, A. M. (2020). (In)satisfação com a imagem corporal e atitudes alimentares em estudantes do ensino secundário. Revista Portuguesa de Enfermagem de Saúde Mental (Spe7), 17-24.
} 


\section{INTRODUÇÃO}

A Organização Mundial da Saúde (2000), tem-se pronunciado sobre a existência de desequilíbrios no comportamento alimentar e da sua estrita relação com a (in)satisfação com a imagem corporal, apelando a uma ideologia de responsabilização individual relativamente às atitudes alimentares dos jovens. Este tema adquire ainda mais pertinência por se tratar de um problema de saúde pública que pode perpetuar-se ao longo de toda a vida.

A imagem corporal é construída por aquilo que o indivíduo vê no espelho e que estrutura mentalmente sobre si mesmo, dependente da aprovação dos "padrões estéticos" (Silva \& Lange, 2010). Outro juízo, intimamente ligado com a imagem corporal é o autoconceito, que se define pelo conhecimento que o próprio indivíduo desenvolve sobre si mesmo. É um conceito multidimensional que pode ser dividido em três componentes básicos: o cognitivo, o afetivo e o comportamental. A componente cognitiva é vista como um conjunto de características que determina a sua personalidade e o seu comportamento, podendo ser ou não real e/ou objetiva. A componente afetiva abrange os sentimentos e as emoções que o próprio individuo sente por si mesmo. A componente comportamental refere-se ao juízo que o individuo faz sobre si mesmo (Martins, Nunes \& Noronha, 2008).

No que se refere aos adolescentes, Fitzgerald, Heary, Kelly e Nixon (2009) referem que os mesmos têm consciência que as escolhas alimentares têm impacto na sua imagem corporal. Segundo estes autores, existem oito fatores que determinam a opção alimentar das crianças e adolescentes, nomeadamente, o conhecimento, as preferências alimentares, as perceções de alimentação saudável, ambiente familiar, influência dos pares, influência da escola, publicidade alimentar e características do estilo de vida. Arcan et al. (2007) reforçam esta ideia, referindo que os jovens ao longo da adolescência alteram as suas dietas e estas podem ser influenciadas por vários fatores. Vaz, Silva, Rego e Viana (2010) referem que o padrão alimentar, que é traduzido pelas atitudes alimentares, é gerado por um processo de desenvolvimento e socialização. Este é o resultado de influências familiares e sociais, desde o seio familiar, conhecimento escolar, acesso à multiculturalidade e nível social.

Existe uma grande pressão sociocultural que estabelece padrões de beleza e ideais de perfeição associados a silhuetas magras.
Nesta linha de pensamento, os adolescentes tendem a procurar alcançar a aparência física perfeita tornando-se um problema quando buscam esse ideal. A troca de hábitos saudáveis por técnicas não saudáveis de controlo de peso pode desencadear distúrbios do comportamento alimentar (Martins et al., 2008). As atitudes alimentares disfuncionais afetam uma percentagem avultada da população adolescente e atualmente começam a manifestar-se em idades mais precoces e, por vezes, permanecem toda a vida, pelo que a deteção precoce e a prevenção desses problemas são de extrema importância e prioridade (Teixeira, 2014).

\section{MÉTODOS}

O estudo efetuado tem uma tipologia observacional, analítica, de paradigma quantitativo, num plano transversal. Para a realização do estudo foi obtida a autorização da Direção do Agrupamento de Escola Emídio Garcia a 7/11/2016, assim como autorização de todos os encarregados de educação para aplicação do instrumento de recolha de dados. A investigação respeita os princípios éticos e pressupostos implícitos na Declaração de Helsínquia e na Convenção de Oviedo.

Para a análise e identificação da satisfação corporal e das atitudes alimentares foi aplicado um questionário que incluía o Questionário de Silhuetas de Collins (Simões, 2014) e a escala Children's Eating Attitude Test (TAAc) validada para a população Portuguesa por Teixeira et al. (2012). O Questionário de Silhuetas de Collins é utilizado para avaliar a (in)satisfação com a imagem corporal das crianças e adolescentes. Esta escala é constituída por uma sequência de sete silhuetas femininas e/ou masculinas, desenvolvidas para ilustrar o peso corporal, alterando da silhueta menos volumosa (1-muito fina) para a mais volumosa (7-obesidade). Relativamente à escala de silhuetas, considera-se a variável classificação da imagem corporal como possibilidade de resposta: satisfeito ou não satisfeito. Na opção satisfeito, estão incluídos os alunos que manifestaram satisfação com o peso na variável imagem corporal e na opção não satisfeito, estão incluídos os alunos que manifestam vontade de ganhar ou perder peso na variável imagem corporal. A discrepância entre a imagem corporal real (imagem que considera mais parecida consigo atualmente) e ideal (imagem que gostaria de ter) é utilizada como indicador de insatisfação com a própria imagem (Simões, 2014). 
O Teste de Atitudes Alimentares para Crianças (TAAc) tem 26 questões e é respondido através de uma escala tipo Likert com seis opções de resposta: "nunca", "raras vezes", "algumas vezes”, "muitas vezes", "muitíssimas vezes" e "sempre”. As respostas sintomáticas são cotadas com (um) “muitas vezes”, (dois) “muitíssimas vezes” e (três) "sempre”. As respostas "nunca", "raras vezes", "algumas vezes" são cotadas com zero (Teixeira, 2014). O TAAc tem dois itens de cotação invertida (19 e 25). A pontuação total do TAAc é calculada somando a pontuação de cada item, quanto mais elevada for a pontuação global mais disfuncionais são as atitudes e comportamentos alimentares. A pontuação total do TAAc é calculada somando a pontuação de cada item, quanto mais elevada for a pontuação global mais disfuncionais são as atitudes e comportamentos alimentares (Teixeira, 2014). Considerou-se o valor igual ou superior a 20, na pontuação total, como ponto de corte para alterações severas do Comportamento Alimentar(CA); Scores $\geq 10<20$ são indicadores de alterações moderadas do CA e Scores $<10$ são indicadores de CA normal.

$\mathrm{O}$ instrumento de recolha de dados foi entregue para preenchimento de forma direta à população da presente investigação, constituída por estudantes do $10 .^{\circ}, 11 .^{\circ}$ e $12 .^{\circ}$ ano de escolaridade de uma escola de Bragança durante o período compreendido entre janeiro e novembro de 2017. A população é constituída por 563 estudantes de ambos os sexos.

A amostra do presente estudo é constituída por 184 adolescentes. Para a investigação optou-se por um processo de amostragem não probabilística, acidental. Os dados foram tratados informaticamente, recorrendo ao programa de tratamento estatístico Statistical Package for the Social Science (SPSS), na versão 22.0. Foram utilizadas frequências absolutas e relativas para descrever as variáveis qualitativas. As variáveis numéricas foram descritas através das medidas: média, desvio padrão, mediana e amplitude interquartil. Na comparação de grupos, atendendo à natureza das variáveis e ao facto de não se ter verificado, através do teste de Kolmogorov-Smirnov, a normalidade das mesmas, utilizaram-se os testes de Mann Whitney e Kruskal Wallis. Para medir a fiabilidade da escala utilizou-se o coeficiente de consistência Alpha de Cronbach. Em todas as análises considerou-se com significância estatística um valor de prova inferior a $5 \%$.

\section{RESULTADOS}

Neste estudo participaram 184 alunos dos quais 45,11\% (83) eram do sexo masculino e 54,89\% (101) do sexo feminino, com idades médias de $16,61 \pm 1,09$ e 16,62 $\pm 0,88$ anos respetivamente. Frequentavam o $10^{\circ}$ ano 33,7\% (62); $33,2 \%$ (61) o $11^{\circ}$ ano e 33,2\% (61) o $12^{\circ}$ ano. Na caraterização da escala TAAc, constata-se uma consistência interna razoável, com exceção da dimensão Controlo da Ingestão Alimentar que apresenta coeficiente Alpha de Cronbach inferior a 0,6. Verifica-se em todas as dimensões da escala TAAc valores médios baixos, assim como os valores da mediana não revelando atitudes e comportamentos alimentares disfuncionais (Tabela 1).

Tabela 1 - Resultados psicométricos obtidos através da aplicação da escala TAAc

\begin{tabular}{|l|l|l|l|l|l|l|}
\hline & Itens & $\begin{array}{l}\text { Alpha de } \\
\text { Cronbach }\end{array}$ & $\begin{array}{l}\text { Amplitude } \\
\text { teórica }\end{array}$ & $\begin{array}{l}\text { Média (desvio } \\
\text { padrão) }\end{array}$ & $\begin{array}{l}\text { Coeficiente } \\
\text { de variação }\end{array}$ & $\begin{array}{l}\text { Mediana (amplitude } \\
\text { interquartil) }\end{array}$ \\
\hline $\begin{array}{l}\text { Aspetos relativos ao cumpri- } \\
\text { mento dieta }\end{array}$ & $\begin{array}{l}1,6,7,10,11, \\
12,14,16,17, \\
22,23,24 \mathrm{e} \\
25\end{array}$ & 0,788 & $0-39$ & $4,10(5,01)$ & $122,30 \%$ & $2(5)$ \\
\hline $\begin{array}{l}\text { Preocupação com comida e } \\
\text { bulimia }\end{array}$ & $\begin{array}{l}3,4,8,18,21 \\
\text { e } 26\end{array}$ & 0,692 & $0-18$ & $1,54(2,62)$ & $170,04 \%$ & $0(2)$ \\
\hline $\begin{array}{l}\text { Controlo da ingestão } \\
\text { alimentar }\end{array}$ & $\begin{array}{l}2,5,9,13,15, \\
19 \mathrm{e} 20\end{array}$ & 0,357 & $0-21$ & $2,97(3,16)$ & $106,17 \%$ & $2(3)$ \\
\hline TAAc & Todos & 0,781 & $0-78$ & $8,61(8,20)$ & $95,30 \%$ & $6(9)$ \\
\hline
\end{tabular}

No que concerne à caraterização dos itens da escala TAAc (Tabela 2), verifica-se que a maioria dos inquiridos afirmaram nunca ou raramente ter os comportamentos abordados, com exceção dos itens: "assusta-me ter peso a mais" em que se destacam as opções raramente $(29,3 \% ; 54)$ e algumas vezes $(21,2 \% ; 39)$; "penso em comida grande parte do dia" onde se destaca as opções raramente (38,0\%; 70) e algumas vezes $(31,5 \%$; 58$)$; 
"corto a minha comida em pequenos pedaços" onde a maioria respondeu raramente $(30,4 \% ; 56)$ ou algumas vezes (30,4\%; 56); "penso em queimar calorias quando faço exercício" com destaque para raramente $(38,0 \% ; 70)$ e algumas vezes $(20,1 \% ; 37)$; "consigo controlar-me com a comida" com frequências mais elevadas em raramente $(27,2 \% ; 51)$ e muitíssimas vezes $(20,7 \% ; 38)$ e "gosto de provar novas comidas apetitosas" em que a maioria respondeu que o faz muitíssimas vezes $(21,7 \% ; 40)$ ou sempre $(32,1 \% ; 59)$.

Tabela 2 - Caraterização dos itens da escala TAAc

\begin{tabular}{|c|c|c|c|c|c|c|c|c|}
\hline \multirow[t]{2}{*}{ Itens da escala } & \multicolumn{8}{|c|}{ Frequência absoluta (frequência relativa \%) } \\
\hline & $\begin{array}{l}\text { Nunca } \\
\mathrm{n}(\%)\end{array}$ & $\begin{array}{l}\text { Raramente } \\
\mathrm{n}(\%)\end{array}$ & $\begin{array}{l}\text { Algumas } \\
\text { Vezes } \\
\mathrm{n}(\%)\end{array}$ & $\begin{array}{l}\text { Muitas } \\
\text { Vezes } \\
\mathrm{n}(\%)\end{array}$ & $\begin{array}{l}\text { Muitíssimas } \\
\text { Vezes } \\
\mathrm{n}(\%)\end{array}$ & $\begin{array}{l}\text { Sempre } \\
\mathrm{n}(\%)\end{array}$ & Mediana & Moda \\
\hline 1.Assusta-me ter peso a mais & $29(15,8 \%)$ & $54(29,3 \%)$ & $39(21,2 \%)$ & $24(13,0 \%)$ & $14(7,6 \%)$ & $24(13,0 \%)$ & 3 & 2 \\
\hline 2.Evito comer quando tenho fome & $56(30,4 \%)$ & $93(51,1 \%)$ & $23(12,5 \%)$ & $5(2,7 \%)$ & $5(2,7 \%)$ & $1(0,5 \%)$ & 2 & 2 \\
\hline 3.Penso em comida grande parte do dia & $10(5,4 \%)$ & $70(38,0 \%)$ & $58(31,5 \%)$ & $12(6,5 \%)$ & $19(10,3 \%)$ & $15(8,2 \%)$ & 3 & 2 \\
\hline $\begin{array}{l}\text { 4. Tem havido vezes em que me sinto } \\
\text { incapaz de parar de comer }\end{array}$ & $30(16,3 \%)$ & $92(50,0 \%)$ & $37(20,1 \%)$ & $12(6,5 \%)$ & $9(4,9 \%)$ & $4(2,2 \%)$ & 2 & 2 \\
\hline $\begin{array}{l}\text { 5. Corto a minha comida em pequenos } \\
\text { pedaços }\end{array}$ & $12(6,5 \%)$ & $56(30,4 \%)$ & $56(30,4 \%)$ & $32(17,4 \%)$ & $11(6,0 \%)$ & $17(9,2 \%)$ & 3 & 2 \\
\hline $\begin{array}{l}\text { 6. Conheço as calorias dos alimentos } \\
\text { que como }\end{array}$ & $31(16,8 \%)$ & $73(39,7 \%)$ & $38(20,7 \%)$ & $20(10,9 \%)$ & $15(8,2 \%)$ & $7(3,8 \%)$ & 2 & 2 \\
\hline $\begin{array}{l}\text { 7. Evito alimentos como pão, batatas } \\
\text { fritas e arroz }\end{array}$ & $41(22,3 \%)$ & $76(41,3 \%)$ & $46(25,0 \%)$ & $18(9,8 \%)$ & $1(0,5 \%)$ & $2(1,1 \%)$ & 2 & 2 \\
\hline $\begin{array}{l}\text { 8. Sinto que os outros gostariam que eu } \\
\text { comesse mais }\end{array}$ & $48(26,1 \%)$ & $67(36,4 \%)$ & $42(22,8 \%)$ & $9(4,9 \%)$ & $11(6,0 \%)$ & $7(3,8 \%)$ & 2 & 2 \\
\hline 9. Vomito depois de comer & $108(58,7 \%)$ & $67(36,4 \%)$ & $5(2,7 \%)$ & $2(1,1 \%)$ & $1(0,5 \%)$ & $1(0,5 \%)$ & 1 & 1 \\
\hline 10. Sinto-me culpado depois de comer & $70(38,0 \%)$ & $84(45,7 \%)$ & $17(9,2 \%)$ & $4(2,2 \%)$ & $8(4,3 \%)$ & $1(0,5 \%)$ & 2 & 2 \\
\hline 11. Penso muito sobre querer ser magro & $34(18,5 \%)$ & $71(38,6 \%)$ & $43(23,4 \%)$ & $15(8,2 \%)$ & $8(4,3 \%)$ & $13(7,1 \%)$ & 2 & 2 \\
\hline $\begin{array}{l}\text { 12. Penso em comer calorias quando } \\
\text { faço exercício }\end{array}$ & $21(11,4 \%)$ & $70(38,0 \%)$ & $37(20,1 \%)$ & $29(15,8 \%)$ & $12(6,5 \%)$ & $15(8,2 \%)$ & 3 & 2 \\
\hline $\begin{array}{l}\text { 13. Os outros pensam que estou muito } \\
\text { magro }\end{array}$ & $43(23,4 \%)$ & $73(39,7 \%)$ & $36(19,6 \%)$ & $14(7,6 \%)$ & $12(6,5 \%)$ & $6(3,3 \%)$ & 2 & 2 \\
\hline $\begin{array}{l}\text { 14. Penso muito se tenho gordura no } \\
\text { meu corpo }\end{array}$ & $27(14,7 \%)$ & $77(41,8 \%)$ & $42(22,8 \%)$ & $17(9,2 \%)$ & $15(8,2 \%)$ & $6(3,3 \%)$ & 2 & 2 \\
\hline $\begin{array}{l}\text { 15. Demoro mais tempo que os outros } \\
\text { a comer as refeições }\end{array}$ & $21(11,4 \%)$ & $76(41,3 \%)$ & $32(17,4 \%)$ & $21(11,4 \%)$ & $11(6,0 \%)$ & $23(12,5 \%)$ & 2 & 2 \\
\hline 16. Evito alimentos com açúcar & $29(15,8 \%)$ & $74(40,2 \%)$ & $46(25,0 \%)$ & $19(10,3 \%)$ & $10(5,4 \%)$ & $6(3,3 \%)$ & 2 & 2 \\
\hline 17. Como comida de dieta & $58(31,5 \%)$ & $81(44,0 \%)$ & $27(14,7 \%)$ & $9(4,9 \%)$ & $9(4,9 \%)$ & $0(0,0 \%)$ & 2 & 2 \\
\hline $\begin{array}{l}\text { 18. Penso que a comida controla a } \\
\text { minha vida }\end{array}$ & $55(29,9 \%)$ & $80(43,5 \%)$ & $25(13,6 \%)$ & $9(4,9 \%)$ & $10(5,4 \%)$ & $5(2,7 \%)$ & 2 & 2 \\
\hline $\begin{array}{l}\text { 19. Consigo controlar-me com a } \\
\text { comida }\end{array}$ & $5(2,7 \%)$ & $51(27,7 \%)$ & $29(15,8 \%)$ & $30(16,3 \%)$ & $38(20,7 \%)$ & $31(16,8 \%)$ & 4 & 2 \\
\hline $\begin{array}{l}\text { 20. Sinto que os outros me pressionam } \\
\text { para comer }\end{array}$ & $46(25,0 \%)$ & $92(50,0 \%)$ & $29(15,8 \%)$ & $8(4,3 \%)$ & $7(3,8 \%)$ & $2(1,1 \%)$ & 2 & 2 \\
\hline $\begin{array}{l}\text { 21. Gasto demasiado tempo a pensar } \\
\text { em comida }\end{array}$ & $42(22,8 \%)$ & $100(54,3 \%)$ & $20(10,9 \%)$ & $8(4,3 \%)$ & $9(4,9 \%)$ & $5(2,7 \%)$ & 2 & 2 \\
\hline $\begin{array}{l}\text { 22. Sinto-me desconfortável depois de } \\
\text { comer doces }\end{array}$ & $59(32,1 \%)$ & $67(36,4 \%)$ & $31(16,8 \%)$ & $13(7,1 \%)$ & $7(3,8 \%)$ & $7(3,8 \%)$ & 2 & 2 \\
\hline 23. Tenho andado a fazer dietas & $66(35,9 \%)$ & $69(37,5 \%)$ & $25(13,6 \%)$ & $14(7,6 \%)$ & $8(4,3 \%)$ & $2(1,1 \%)$ & 2 & 2 \\
\hline $\begin{array}{l}24 \text {. Gosto de sentir o meu estômago } \\
\text { vazio }\end{array}$ & $79(42,9 \%)$ & $76(41,3 \%)$ & $18(9,8 \%)$ & $6(3,3 \%)$ & $4(2,2 \%)$ & $1(0,5 \%)$ & 2 & 1 \\
\hline 25. Gosto de provar comidas apetitosas & $3(1,6 \%)$ & $31(16,8 \%)$ & $21(11,4 \%)$ & $30(16,3 \%)$ & $40(21,7 \%)$ & $59(32,1 \%)$ & 5 & 6 \\
\hline $\begin{array}{l}\text { 26. Tenho impulso de vomitar depois } \\
\text { de comer }\end{array}$ & $104(56,5 \%)$ & $65(35,3 \%)$ & $9(4,9 \%)$ & $4(2,2 \%)$ & $1(0,5 \%)$ & $1(0,5)$ & 1 & 1 \\
\hline
\end{tabular}


Verifica-se ainda que as atitudes alimentares são estatisticamente distintas entre os rapazes e as raparigas e que os alunos do $11^{\circ}$ ano apresentaram resultados mais elevados, quer em termos médios quer em termos de dispersão de resultados, na dimensão aspetos relativos ao cumprimento da dieta (média de 4,66 $\pm 6,21$ ); na dimensão controlo da

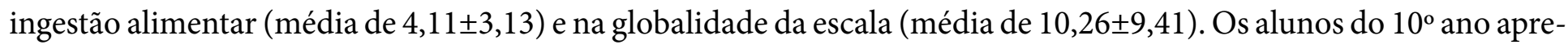
sentaram os resultados mais baixos na dimensão aspetos relativos ao cumprimento da dieta (média de 3,58 $\pm 4,03$ ), na

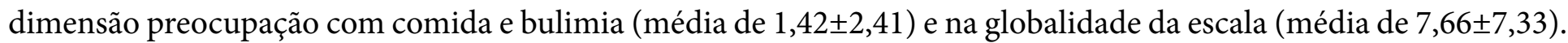
As diferenças observadas são estatisticamente significativas na dimensão controlo da ingestão alimentar (Tabela 3).

Tabela 3 - Caracterização da Escala TAAc em função das variáveis Sexo e Ano de Escolaridade

\begin{tabular}{|l|l|l|l|l|l|}
\hline Variáveis & Opção & $\begin{array}{l}\text { Aspetos relativos ao } \\
\text { cumprimento dieta }\end{array}$ & $\begin{array}{l}\text { Preocupação com } \\
\text { comida e bulimia }\end{array}$ & $\begin{array}{l}\text { Controlo da ingestão } \\
\text { alimentar }\end{array}$ & TAAc \\
\hline \multirow{5}{*}{ Sexo } & $\begin{array}{l}\text { Masculino } \\
\text { Média (d. padrão) }\end{array}$ & $3,33(4,60)$ & $1,47(2,76)$ & $2,76(3,47)$ & $7,55(7,91)$ \\
\cline { 2 - 6 } & $\begin{array}{l}\text { Feminino } \\
\text { Média (d. padrão) }\end{array}$ & $4,73(5,26)$ & $1,59(2,51)$ & $3,15(2,88)$ & $9,48(8,38)$ \\
\cline { 2 - 6 } & $\begin{array}{l}\text { Mann-Whitney } \\
\text { Valor prova (p) }\end{array}$ & $0,027^{\star}$ & 0,286 & 0,112 & $0,026^{\star}$ \\
\hline Ano de escolaridade & $\begin{array}{l}10^{\circ} \text { ano } \\
\text { Média (d. padrão) }\end{array}$ & $3,58(4,03)$ & $1,42(2,41)$ & $2,66(3,52)$ & $7,66(7,33)$ \\
\cline { 2 - 6 } & $\begin{array}{l}11^{\circ} \text { ano } \\
\text { Média (d. padrão) }\end{array}$ & $4,66(6,21)$ & $1,49(2,13)$ & $4,11(3,13)$ & $10,26(9,41)$ \\
\cline { 2 - 6 } & $\begin{array}{l}12^{\circ} \text { ano } \\
\text { Média (d. padrão) }\end{array}$ & $4,07(4,58)$ & $1,70(3,22)$ & $2,15(2,43)$ & $7,92(7,61)$ \\
\cline { 2 - 6 } & $\begin{array}{l}\text { Kruskal Wallis } \\
\text { Valor prova (p) }\end{array}$ & 0,732 & 0,797 & $0,000^{* *}$ & 0,212 \\
\hline
\end{tabular}

${ }^{*}$ significativo a $5 \% ;{ }^{* *}$ significativo a $1 \%$

Após determinação da satisfação da imagem corporal através da diferença entre a silhueta atual e a silhueta desejada, verifica-se que $14,1 \%$ dos alunos inquiridos manifestaram desejo de ganhar peso, por outro lado, $47,8 \%$ deseja perder peso. Constata-se que $38 \%$ dos inquiridos referiu estar satisfeito com a imagem corporal atual (Tabela 4).

Tabela 4 - Caracterização da Escala TAAc em função das variáveis Sexo e Ano de Escolaridade

\begin{tabular}{|c|c|c|c|c|}
\hline \multicolumn{2}{|l|}{ Variáveis } & \multicolumn{2}{|c|}{ Sexo } & \multirow[b]{2}{*}{$\begin{array}{l}\text { Total } \\
\text { n(\%linha) } \\
\text { frequência } \\
\text { relativa \% }\end{array}$} \\
\hline & & $\begin{array}{l}\text { Masculino } \\
\mathrm{n}(\% \text { linha }) \\
\text { frequência } \\
\text { relativa \% }\end{array}$ & $\begin{array}{l}\text { Feminino } \\
\mathrm{n}(\% \text { linha }) \\
\text { frequência } \\
\text { relativa \% }\end{array}$ & \\
\hline \multirow{6}{*}{$\begin{array}{l}\text { Imagem } \\
\text { corporal }\end{array}$} & \multirow{2}{*}{$\begin{array}{l}\text { Ganho de } \\
\text { peso }\end{array}$} & $12(46,2 \%)$ & $14(53,8 \%)$ & $26(100 \%)$ \\
\hline & & $14,4 \%$ & $13,9 \%$ & $14,1 \%$ \\
\hline & \multirow{2}{*}{$\begin{array}{l}\text { Satisfação } \\
\text { com peso }\end{array}$} & $35(50,0 \%)$ & $35(50,0 \%)$ & $70(100 \%)$ \\
\hline & & $42,2 \%$ & $34,6 \%$ & $38,0 \%$ \\
\hline & \multirow{2}{*}{$\begin{array}{l}\text { Perda de } \\
\text { peso }\end{array}$} & $36(40,9 \%)$ & $52(59,1 \%)$ & $88(100 \%)$ \\
\hline & & $43,4 \%$ & $51,5 \%$ & $47,8 \%$ \\
\hline \multirow{2}{*}{\multicolumn{2}{|c|}{ Total }} & $83(45,1 \%)$ & $101(54,9 \%)$ & $184(100 \%)$ \\
\hline & & $100 \%$ & $100 \%$ & $100 \%$ \\
\hline
\end{tabular}

Verificou-se ainda que a satisfação com a silhueta não estava significativamente associada ao sexo do aluno em estudo, nem ao ano de escolaridade (Tabela 5).

Tabela 5 - Caracterização da variável Satisfação com a Imagem Corporal em função das variáveis Sexo e Ano de Escolaridade

\begin{tabular}{|c|c|c|c|c|c|}
\hline \multirow{2}{*}{\multicolumn{2}{|c|}{ Variáveis }} & \multicolumn{2}{|c|}{ Classificação Silhuetas } & \multirow[b]{2}{*}{$\begin{array}{l}\text { Total } \\
\text { n(\%linha) } \\
\text { frequência } \\
\text { relativa \% }\end{array}$} & \multirow[b]{2}{*}{$\begin{array}{l}\text { Qui-quadra- } \\
\text { do } \\
\text { Estatística } \\
\text { teste } \\
\text { (valor de } \\
\text { prova) }\end{array}$} \\
\hline & & $\begin{array}{l}\text { Satisfeito } \\
\mathrm{n}(\% \text { linha }) \\
\text { frequência } \\
\text { relativa \% }\end{array}$ & $\begin{array}{l}\text { Não Satis- } \\
\text { feito } \\
\text { n(\%linha) } \\
\text { frequência } \\
\text { relativa \% }\end{array}$ & & \\
\hline \multirow[t]{6}{*}{ Sexo } & \multirow{2}{*}{$\begin{array}{l}\text { Mascu- } \\
\text { lino }\end{array}$} & $35(42,2 \%)$ & $48(57,8 \%)$ & $83(100 \%)$ & \multirow[t]{4}{*}{$1,092(0.296)$} \\
\hline & & $50,0 \%$ & $42,1 \%$ & $45,1 \%$ & \\
\hline & \multirow{2}{*}{$\begin{array}{l}\text { Femi- } \\
\text { nino }\end{array}$} & $35(34,7 \%)$ & $66(65,3 \%)$ & $101(100 \%)$ & \\
\hline & & $50,0 \%$ & $57,9 \%$ & $54,9 \%$ & \\
\hline & \multirow[t]{2}{*}{ Total } & $70(38,0 \%)$ & $114(62,0 \%)$ & $184(100 \%)$ & \\
\hline & & $100 \%$ & $100 \%$ & $100 \%$ & \\
\hline \multirow{6}{*}{$\begin{array}{l}\text { Ano de } \\
\text { escolari- } \\
\text { dade }\end{array}$} & \multirow[t]{2}{*}{$10^{\circ}$. ano } & $25(40,3 \%)$ & $37(59,7 \%)$ & $62(100 \%)$ & \multirow[t]{6}{*}{$1,075(0,584)$} \\
\hline & & $35,7 \%$ & $32,5 \%$ & $33,7 \%$ & \\
\hline & \multirow[t]{2}{*}{$11 .^{\circ}$ ano } & $25(41,0 \%)$ & $36(59,0 \%)$ & $61(100 \%)$ & \\
\hline & & $35,7 \%$ & $31,6 \%$ & $33,2 \%$ & \\
\hline & \multirow[t]{2}{*}{$12 .^{\circ}$ ano } & $20(32,8 \%)$ & $41(67,2 \%)$ & $61(100 \%)$ & \\
\hline & & $28,6 \%$ & $36,0 \%$ & $33,2 \%$ & \\
\hline \multirow{2}{*}{\multicolumn{2}{|c|}{ Total }} & $83(45,1 \%)$ & $101(54,9 \%)$ & $184(100 \%)$ & \\
\hline & & $100 \%$ & $100 \%$ & $100 \%$ & \\
\hline
\end{tabular}


A análise dos resultados do que se refere à relação entre as atitudes alimentares e o grau de (in)satisfação com a imagem corporal em estudantes do ensino secundário (Tabela 6), permite constatar que os jovens não satisfeitos com a sua silhueta apresentaram resultados mais elevados nos aspetos relativos ao cumprimento da dieta $(4,69 \pm 5,19)$; no controlo da ingestão alimentar $(2,62 \pm 2,83)$ e na globalidade da TAAc $(8,79 \pm 8,32)$. Na dimensão preocupação com comida e bulimia foram os alunos satisfeitos com a imagem que apresentaram maior valor médio e menor dispersão $(1,64 \pm 2,53)$. As diferenças observadas revelaram que a dimensão cumprimento da dieta e controlo da ingestão alimentar são estatisticamente distintas entre os jovens satisfeitos e não satisfeitos com a sua silhueta.

Tabela 6 - Caracterização da escala TAAc em função da variável Satisfação com a Imagem Corporal

\begin{tabular}{|l|l|l|l|l|l|}
\hline Variáveis & Opção & $\begin{array}{l}\text { Aspetos relativos ao } \\
\text { cumprimento dieta }\end{array}$ & $\begin{array}{l}\text { Preocupação com } \\
\text { comida e bulimia }\end{array}$ & $\begin{array}{l}\text { Controlo da ingestão } \\
\text { alimentar }\end{array}$ & TAAc \\
\hline $\begin{array}{l}\text { Satisfação com } \\
\text { imagem } \\
\text { corporal }\end{array}$ & $\begin{array}{l}\text { Satisfeito } \\
\text { Média(d. padrão) }\end{array}$ & $3,13(4,57)$ & $1,64(2,53)$ & $2,54(3,57)$ & $8,31(8,07)$ \\
\cline { 2 - 6 } & $\begin{array}{l}\text { Não Satisfeito } \\
\text { Média(d. padrão) }\end{array}$ & $4,69(5,19)$ & $1,47(2,68)$ & $2,62(2,83)$ & $8,79(8,32)$ \\
\cline { 2 - 6 } & $\begin{array}{l}\text { Mann-Whitney } \\
\text { Valor prova (p) }\end{array}$ & $0,004^{* *}$ & 0,330 & $0,033^{*}$ & 0,638 \\
\hline
\end{tabular}

${ }^{\star}$ significativo a $5 \% ;{ }^{* *}$ significativo a $1 \%$

\section{DISCUSSÃO}

No trabalho atual encontraram-se valores médios baixos, assim como os valores da mediana, em todas as dimensões da escala TAAc, o que se traduz em atitudes alimentares normais. Altos índices de atitudes alimentares foram encontrados no estudo de Krepp e Ferreira (2012), tal como no estudo de Teixeira (2014), realizado em Coimbra, onde numa amostra de 978 adolescentes, 12,33\% apresentava atitudes alimentares disfuncionais. Comparativamente aos estudos supracitados, o estudo atual apresentou resultados indicativos de atitudes alimentares com valores de média e moda mais baixos.

Verifica-se ainda que as atitudes alimentares são estatisticamente distintas entre os rapazes e as raparigas. Estes resultados são corroborados na literatura, onde se constata que as atitudes alimentares com valores mais elevados e consequentemente mais disfuncionais, são mais frequentes no sexo feminino (Gonzáles-Juárez et al., 2007; Maor, Savag, Dahan \& Hermoni, 2006; Vilela, Lamounier, Dellaretti Filho, Barros Neto \& Horta, 2004).

No que diz respeito à satisfação com a imagem corporal, Zordão et al. (2015), referem que na adolescência a imagem corporal é frequentemente idealizada, não correspondendo à imagem real. A perspetiva ideal de beleza, muitas vezes associada ao sucesso, difundida pelos meios de comunicação social, pode desencadear um processo de negação culminando com a insatisfação corporal. No presente estudo, $62 \%$ dos adolescentes não estavam satisfeitos com a sua imagem corporal, observando-se ainda que a satisfação com a silhueta não estava significativamente associada ao sexo. Outros estudos têm concluído que jovens do sexo feminino apresentam maior insatisfação com a imagem corporal quando comparadas aos rapazes (Teixeira, 2014). No estudo de Marques et al. (2016), foram inquiridos 223 jovens, com idades compreendidas entre os 12 e os 19 anos constatando-se que $64,6 \%$ dos jovens estavam insatisfeitos com a imagem corporal, com uma maior prevalência para o sexo feminino. Os dados nacionais da saúde dos adolescentes portugueses (Health Behaviour in School - Aged Children (HBSC) 2014) apresenta valores mais baixos, onde $53,4 \%$ dos adolescentes afirmaram que não estavam a fazer dieta, porque estão satisfeitos com o seu peso. Os rapazes consideram mais frequentemente ter um corpo ideal.

No que respeita aos dados da TAAc relativamente à satisfação com a imagem corporal as diferenças observadas apresentaram evidência estatística na dimensão cumprimento da dieta e controlo da ingestão alimentar. 
Laus, Costa e Almeida (2011), desenvolveram um estudo com o objetivo de avaliar as relações entre insatisfação com a aparência e atitudes alimentares em adolescentes concluindo que indivíduos insatisfeitos com a aparência têm preocupações anormais com relação à alimentação e peso, independentemente do gênero. No estudo efetuado por Krepp e Ferreira (2012), os resultados demonstraram um significativo número de adolescentes que apresentavam comportamentos alimentares de risco e uma alta prevalência de distorção corporal grave e moderada, observando que as adolescentes que apresentam distorção de imagem corporal possuem um risco significativo de ter um distúrbio alimentar, independente do grau de distorção. Ao examinar a escala TAAc verificou-se que os alunos do $11^{\circ}$ ano apresentaram resultados mais elevados na globalidade da escala. No que concerne a este aspeto, Barry e Lipmann (1990) referem que a prevalência das distorções alimentares é variável consoante a idade. Os estudos de Teixeira (2008) e de Miotto, De Coppi, Freza e Preti (2003) corroboram o nosso estudo, constatando-se que a faixa etária dos 16-17 anos tinha valores mais elevados nos testes de atitudes alimentares.

\section{CONCLUSÃO}

Os adolescentes encontram-se na sua maioria (62\%) insatisfeitos com a imagem corporal, apesar de se verificar em todas as dimensões da escala TAAc, valores médios e da mediana baixos, não revelando atitudes e comportamentos alimentares disfuncionais.

As atitudes alimentares são diferentes entre os jovens satisfeitos e os não satisfeitos com a sua imagem corporal. Os resultados realçam a necessidade da implementação de estratégias e intervenções socioeducativas ao nível dos fatores que conduzem à insatisfação com a imagem corporal. Esta intervenção deve ser alargada à família, tendo em consideração que os primeiros anos de vida são fundamentais para o crescimento físico, desenvolvimento psicossocial, construção de identidade, e por consequentemente, para a aquisição de hábitos alimentares, que, de acordo com as atitudes e comportamentos da família, podem definir os futuros padrões de consumo. Como limitações deste estudo referimos o facto de não ter sido solicitado parecer de uma Comissão de Ética.

\section{REFERÊNCIAS BIBLIOGRÁFICAS}

Arcan, C., Neumark-Sztainer, D., Hannan, P., Van Den Berg, P., Story, M. \& Larson, N. (2007). Parental eating behaviours, home food environment and adolescent intakes of fruits, vegetables and dairy foods: longitudinal findings from Project EAT. Public Health Nutrition, 10(11), 1257-65. Disponível em: https:// www.ncbi.nlm.nih.gov/pubmed/17391551

Barry, A., \& Lipmann, B.B. (1990). Anorexia nervosa in males. Postgraduate Medicine, 24, 12-18. Doi: 10.1080/00325481.1990.11704680

Fitzgerald, A., Heary, C., Kelly, C. \& Nixon, E. (2009). Factors influencing the food consumption of children and adolescents: a qualitative investigation. Proceedings of the Nutrition Society, 67 (OCE7), E226. Doi: $10.1017 /$ S0029665108008896

Gonzáles-Juárez, C., Pérez-Perez, E., Martín Cabrera, B., Mitja Pau, I., Roy de Pablo, R., \& Vazquez de la Torre Escalera, P. (2007). Detection of Adolescents at Risk of Suffering Eating Disorders. Atencion Primaria, 39(4), 189-94. Disponível em: https://www. ncbi.nlm.nih.gov/pubmed/17428423

Health Behaviour in School - Aged Children (HBSC ) (2014). A Saúde dos Adolescentes Portugueses em Tempos de Recessão. Dados Nacionais 2014. Disponível em: http://aventurasocial.com/arquivo/1437158618_RELATORIO\%20HBSC\%202014e. pdf

Krepp, A.C.M. \& Ferreira, G. (2012). Body Dissatisfaction and Eating Habits of Adolescents in a Private School in a Municipality in Southern Minas Gerais. Revista Ciências em Saúde, 2(4), 42-50. Disponível em: https://www.locus.ufv.br/bitstream/handle/123456789/17725/artigo.pdf? sequence $=1$

Laus, M.F., Costa, T.M.B., \& Almeida, S.S. (2011). Body image dissatisfaction and its relationship with physical activity and body mass indez in Brazilian adolescents. Jornal Brasileiro de Psiquiatria, 60(4), 315320. Doi: 10.1590/S0047-20852011000400013 
Maor, N.R., Savag, S., Dahan, R., \& Hermoni, D. (2006). Eating Attitudes among Adolescents. Israel Medical Association Journal, 8(9), 627-9. Disponível em: https://www.ncbi.nlm.nih.gov/pubmed/17058414

Marques, M.I., Pimenta, J., Reis, S., Ferreira, L.M., Peralta. L., Santos, M.I. Santos, S., \& Santos, E. (2016). (In)Satisfação com a imagem corporal na adolescência. Nascer e Crescer, 25(.4), 217-221. Disponível em: http://www.scielo.mec.pt/scielo.php?script=sci_ abstract\&pid $=$ S0872-07542016000600004\&lng $=$ pt \& nrm=iso

Martins, D.F., Nunes, M.F.O., \& Noronha, A.P.P. (2008). Satisfação com a imagem corporal e autoconceito em adolescentes. Psicologia: Teoria e Prática, 10(2), 94-105. Disponível em: http://pepsic.bvsalud. org/pdf/ptp/v10n2/v10n2a08.pdf

Miotto, P., De Coppi, M., Freza, M., \& Preti, A. (2003). The Spectrun of Eating Disorders: Prevalence in an Area of Northeast Italy. Psychiatr Research, 119, 14554. Doi: 10.1016/S0165-1781(03)00128-8

Organização Mundial da Saúde (2000). Obesity: Preventing and Managing the Global Epidemic, OMS Consultation on Obesity. Disponível em: https:// www.who.int/nutrition/publications/obesity/WHO_ TRS_894/en/

Silva, G.A. \& Lange, E.S.N. (2010). Imagem Corporal: A perceção do conceito em indivíduos obesos do sexo feminino. Psicologia Argumento, 28(60), 43-54. Disponível em: https://periodicos.pucpr.br/index.php/ psicologiaargumento/article/view/19779

Simões, A. F. S. (2014). Avaliação da (In)satisfação com a Imagem Corporal: Estudo de Validação da Escala de Silhuetas de Collins para Crianças e Adolescentes Portugueses. (Dissertação de mestrado, Universidade de Coimbra, Portugal). Disponível em: https://estudogeral.sib.uc.pt/handle/10316/27202

Teixeira, M.C.B, Pereira, A.T.F., Saraiva, J. M. T., Marques, M., Soares, M. J., Vaz, S. C., Valente, J., Azevedo, M. H, P. \& Macedo, A. J. F. (2012). Portuguese Validation of the Children's Eating Attitudes Test. Revista Psiquiatria Clínica, 39(6), 189-93. Doi: 10.1590/ S0101-60832012000600002
Teixeira, M.C.B. (2008). Estudo de Atitudes e Comportamentos Alimentares numa População Adolescente. (Dissertação de Mestrado em Nutrição Clínica, Universidade de Coimbra, Portugal). Disponível em: https://estudogeral.uc.pt/handle/10316/18109

Teixeira, M.C.B. (2014). Atitudes e Comportamentos Alimentares numa População Adolescente: $\mathrm{O}$ Papel do Perfecionismo. (Tese de Doutoramento em Ciências da Saúde, Universidade de Coimbra, Portugal). Disponível em https://estudogeral.sib.uc.pt/ handle/10316/25856

Vaz, A., Silva, D., Rego, C. \& Viana, V. (2010). Determinantes comportamentais em crianças e adolescentes com diagnóstico de obesidade. Alimentação Humana. Revista da SPCNA, 16(2), 31-36. Disponível em: https://repositorio-aberto.up.pt/handle/10216/52557

Vilela, J.E.M., Lamounier, J.A., Dellaretti Filho, M.A., Barros Neto, J. \& Horta, G.M. (2004). Transtornos alimentares em escolares. Jornal de Pediatria, 80(1), 49-54. Disponível em: http://www.scielo.br/pdf/jped/ v80n1/v80n1a10

Zordão, O.P., Barbosa, A., Parisi, T. S., Grasselli, C.S.M., Nogueira, D.A., \& Silva, R.R. (2015). Associação da imagem corporal e transtornos alimentares em adolescentes em Minas Gerais. Nutrición Clínica y Dietética Hospitalaria, 35 (2), 48-56. Disponível em: https://dialnet.unirioja.es/servlet/ articulo? codigo $=5137739$

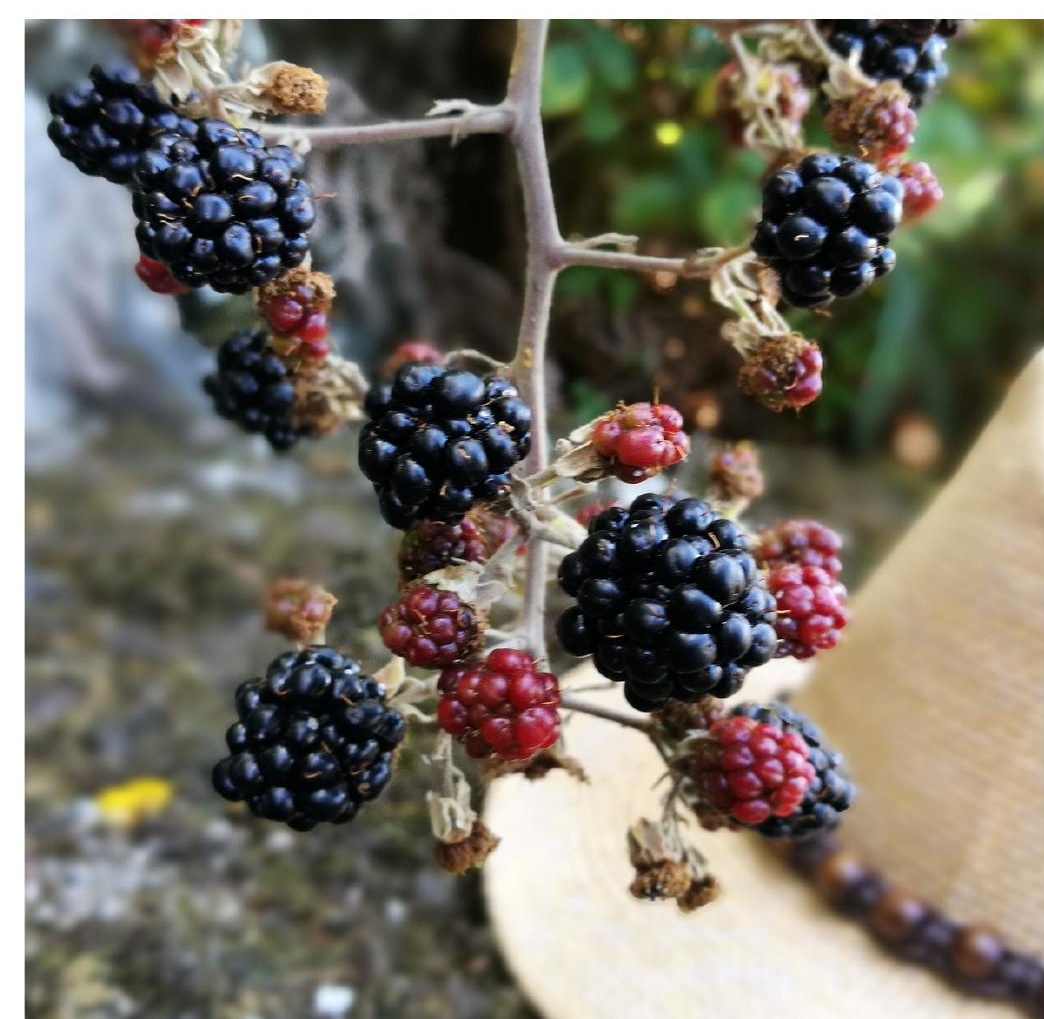

